\title{
A single-channel microparticle sieve based on Brownian ratchets $\uparrow$
}

\author{
Simon Verleger, ${ }^{* a}$ Andrej Grimm ${ }^{b}$ Christian Kreuter, ${ }^{a}$ Huei Ming Tan, ${ }^{c}$ Jeroen A. van Kan, ${ }^{d}$ Artur Erbe, ${ }^{e}$ \\ Elke Scheer ${ }^{a}$ and Johan R. C. van der Maarel ${ }^{b}$
}

DOI: $10.1039 / \mathrm{c} 2 \mathrm{lc} 21089 \mathrm{~d}$

\begin{abstract}
We present a novel device for the separation of microparticles in a single channel, which is made of inversely asymmetric Brownian ratchets. It enables separation into two different fractions with an adjustable threshold and can be modeled with good agreement. This device serves as proof of concept for an extremely compact class of microsieves.
\end{abstract}

The integration of microparticle separators into lab-on-a-chip devices is a crucial issue for the ongoing development in the field of microfluidics. ${ }^{1,2}$ Preferably, such a separator would exhibit a channel structure to easily integrate with other microfluidic components. In order to achieve lab-on-a-chip devices with small overall dimensions and to enable versatile designs of microfluidic circuits, the construction of compact separators is essential. Previously, various separation mechanisms have been demonstrated, ${ }^{3,4}$ such as hydrodynamic filtration, ${ }^{5}$ electrophoresis, ${ }^{6}$ magnetophoresis, ${ }^{7}$ acoustophoresis ${ }^{8}$ and separation through optical forces. ${ }^{9}$ Further, Brownian ratchets have been identified as a concept to transport microparticles, ${ }^{10-13}$ and have been suggested ${ }^{14-18}$ and implemented ${ }^{19-22}$ as separators before. However, none of the before-mentioned techniques features the desired compactness. This article presents a new class of extremely compact microsieves. By performing the separation in a single channel, our device features dimensions which are about one order of magnitude smaller than the smallest separator fabricated by other methods to date.

We schematically illustrate our separation method in Fig. 1. The device consists of a single channel made of two opposing sawtoothshaped walls. A periodically inverting external force $F_{\mathrm{ext}}$ is applied to the particles perpendicular to the channel ( $y$-direction). We consider two particles with distinct drift velocities, i.e., a slow particle (blue)

"Fachbereich Physik, University Konstanz, D-78457 Konstanz, Germany. E-mail: simon.verleger@uni-konstanz.de

${ }^{b}$ Department of Physics, Biophysics and Complex Fluids Group, National University of Singapore, 2 Science Drive 3, Singapore 117542

Engineering Science Programme, National University of Singapore, 9 Engineering Drive 1, Singapore 117576

"Department of Physics, Center for Ion Beam Applications, National University of Singapore, 2 Science Drive 3, Singapore 117542

"Helmholtz-Zentrum Dresden-Rossendorf, FWIO, D-01328 Dresden, Germany

$\dagger$ Electronic supplementary information (ESI) available: Video of the separation of $3.0 \mu \mathrm{m} / 4.3 \mu \mathrm{m}$ colloids, Materials and Methods, Fig. S1-S3. See DOI: $10.1039 / \mathrm{c} 21 \mathrm{c} 21089 \mathrm{~d}$

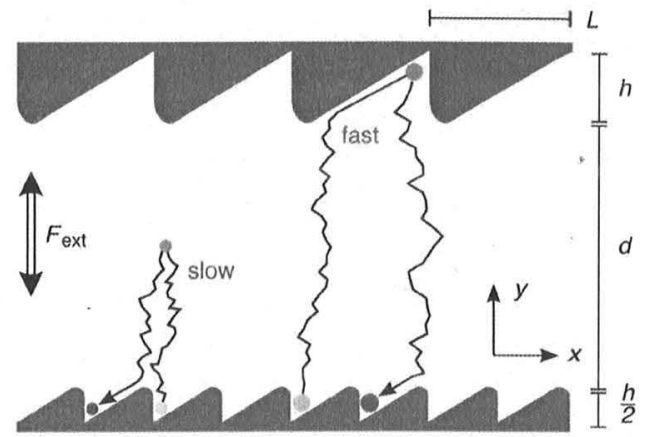

Fig. 1 Schematic top view on the channel separator, showing a full separation cycle. A periodically inverting external force $F_{\text {ext }}$ drags two diffusive particles featuring different drift velocities in the $\pm y$-direction, starting from the small saw teeth. Possible diffusion paths are schematically depicted. Channel width $d$, large sawtooth length $L$, and profile amplitudes $h$ and $h / 2$ are indicated.

and a fast particle (red). While crossing the channel under the influence of the force, both particles are subjected to Brownian motion. Each time a particle arrives at either side of the channel and slides along the walls, it is laterally displaced in the $x$-direction. The large sawtooth profile with a spatial period of $L$ is designed such that particles are on average displaced towards the right $(+x)$, while the small profile with half the spatial period $L / 2$ induces a mean displacement to the left $(-x)$. A detailed discussion of the underlying ratchet effect can be found in the ESI $\dagger$ (ref. 28-30).

In order to separate both particles along the channel, the switching of the external force needs to be adjusted properly. The trick is to reverse the external force such that both particles arrive at the small profile, but only the fast particle reaches the large profile on the other side of the channel. In this case, the slow particle interacts only with the small profile and is therefore effectively transported towards the left. In contrast, the fast particle interacting with both profiles is transported towards the right, as the large profile induces a larger mean displacement than the small one, due to the $2: 1$ ratio of their spatial periods. As a result, fast and slow particles are transported towards opposite directions along the channel and eventually separate. The speed of the induced transport along the channel is determined by the overall mean displacement $\langle\Delta x\rangle$ after a complete up/ down cycle of the external force. The described separation mechanism is illustrated by Movie S1 $\uparrow$, showing the separation of colloids of $3.0 \mu \mathrm{m}$ and $4.3 \mu \mathrm{m}$ in diameter. 
For the experimental setup, we used gravitation as the external force by periodically tilting the device. The channel was made of polydimethylsiloxane (PDMS), which had been cast into a mold predefined by optical lithography. We then placed the PDMS channel in a glass dish (Fig. 2A), which we filled afterwards with the colloid suspension, sealed with a cover glass, and finally placed on a tiltable stage (Fig. 2B). The stage is mounted on a pendulum, along with LED illumination, a CCD camera, and optics. The pendulum is suspended in its center of mass and driven by a stepper motor. The inclination $\alpha$ of the stage determines the effective gravitational driving force perpendicular to the channel walls, thus $F_{\mathrm{cxt}}=4 / 3\left[\left(\rho_{\mathrm{s}}-\rho_{\mathrm{l}}\right)\right.$ $\left.\pi R^{3} g \sin \alpha\right],{ }^{23}$ with $\rho_{\mathrm{s}}$ and $\rho_{1}$ being the mass density of the solid colloids and the liquid medium, respectively, $R$ the colloid radius, and $g$ the gravitational acceleration. Since the frictional coefficient for a spherical particle is given by $\gamma=6 \pi \eta R$, with $\eta$ being the viscosity of the medium, the resulting drift velocity is proportional to $R^{2}$. We chose

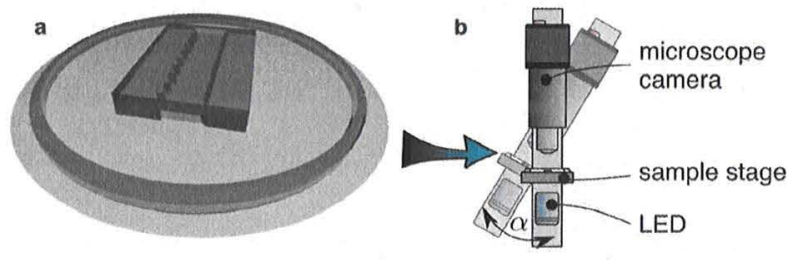

Fig. 2 (a) Sketch of the sample design, dimensions not true to scale. A PDMS block containing the ratchet structure is placed face-down on a cover glass. The suspension is constrained by a glass ring. The cell is sealed by a top cover glass (not shown), resulting in a sample similar to a snow globe. (b) Sketch of the experimental setup: the sample is placed between a camera and an LED on a tiltable rail. silica colloids with mass density $\rho_{\mathrm{s}}=2.0 \mathrm{~g} \mathrm{~cm}^{-3}$ as test particles in water. For efficient separation, the spatial period of the sawtooth profile $L$ has to be notably larger than the particle radius. ${ }^{24}$ The amplitude $h$ should be chosen small, so not to hinder diffusion while at the same time providing sufficient slope for the particles to slide down to the minima. The distance $d$ must be chosen large enough to result in a sufficient spatial margin between the two types of particles, by the time the faster type reaches the large profile. The choice of $L=$ $60 \mu \mathrm{m}, h=40 \mu \mathrm{m}$ and $d=150 \mu \mathrm{m}$ (cf. Fig. 1) was found suitable to match these criteria (see ESI $\dagger$ ).

In order to demonstrate separation in reduced dimensions, we consider a closed channel with ten spatial periods. Fig. 3 shows such a device filled with a suspension of $3.0 \mu \mathrm{m}$ and $4.3 \mu \mathrm{m}$ colloids before (panel A) and after (panel B) the separation process. After 450 cycles, the suspension was fully separated, i.e., $93 \%$ of all particles have reached the two outmost ratchet units on either end. This clearly indicates the excellent binary resolution, i.e., the splitting in two distinct fractions. Note that small differences in particle speeds could be compensated by a larger distance $d$. The time intervals $T_{+}$and $T_{-}$ during which the external force pointed towards $+y$ and $-y$, respectively, were set to $T_{+}=70 \mathrm{~s}$ and $T_{-}=110 \mathrm{~s}$, yielding a duration of 26 hours for the whole process. As discussed later, the duration can be reduced by several orders of magnitude.

For the sake of a more quantitative understanding of the transport properties, we measured the mean displacement for individual particles. For a given particle type, an increase of $T_{+}$is expected to result in a sudden change from negative to positive mean displacement $\langle\Delta x\rangle$, starting as soon as $T_{+}$is sufficiently long for the particles to reach the large profile. With the drift velocity $v_{\text {drift }}$, this threshold is given by $t_{\text {cross }}=d / v_{\text {drift }}$, which denotes the time required to cross the

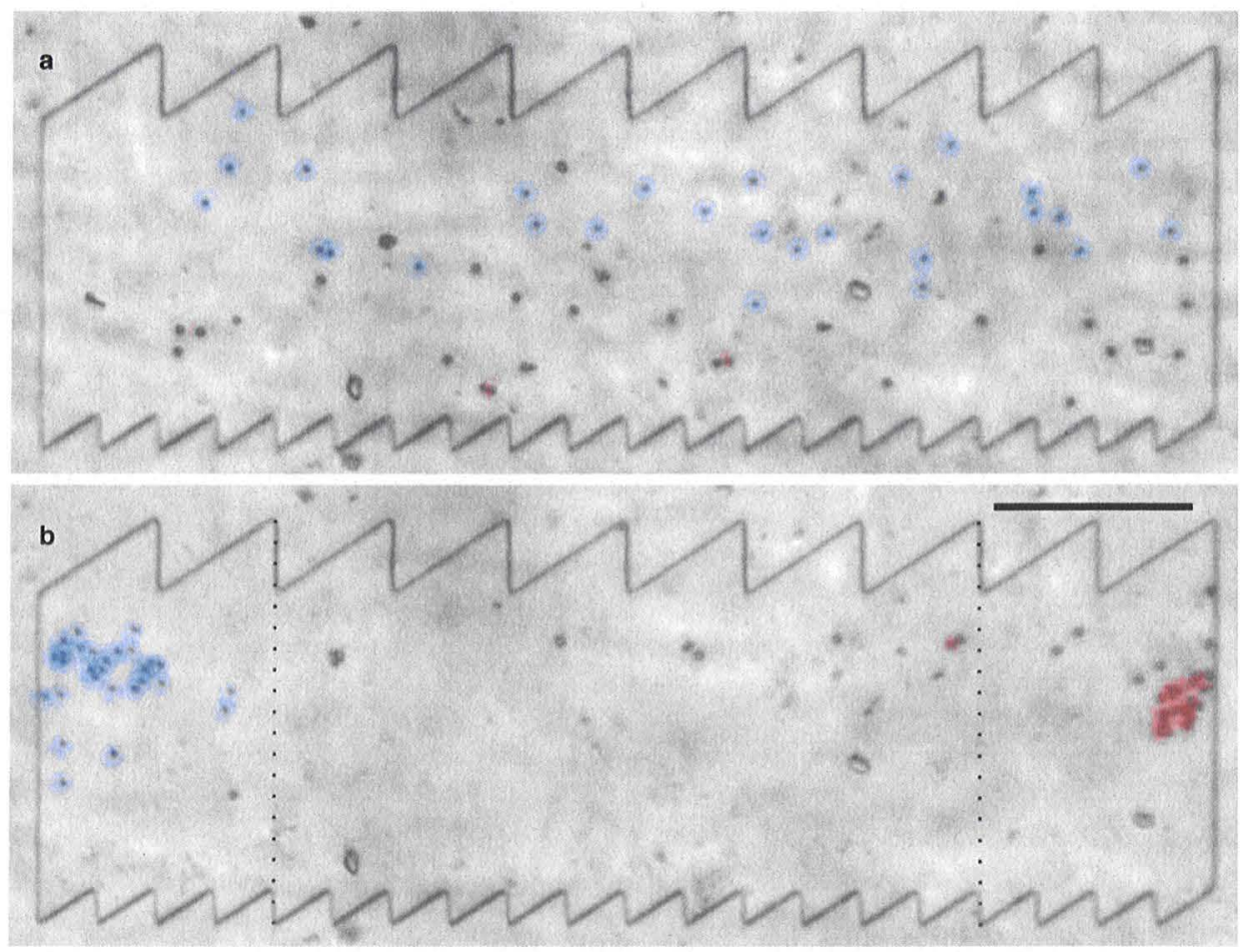

Fig. 3 Photographs of a microsieve before (a) and after (b) the sorting process demonstrate the excellent binary resolution. The closed channel is filled with $3.0 \mu \mathrm{m}$ and $4.3 \mu \mathrm{m}$ silica colloids (highlighted in blue and red, resp.). Scale bar $100 \mu \mathrm{m}$. A similar device in motion is presented online in Movie S1 $\dagger$. 


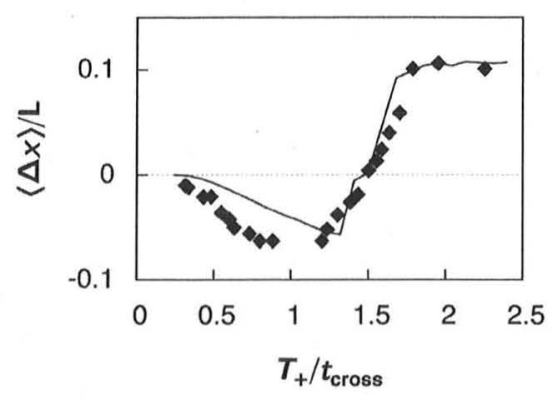

Fig. 4 Control of the transport direction. Mean displacement $\langle\Delta x\rangle / L$ of $3.0 \mu \mathrm{m}$ colloids $v s$. time $T_{+} / t_{\text {cross }}$. Each data point represents $\langle\Delta x\rangle / L$ after 100 cycles for a given $T_{+}$. The experimental data (diamonds) are modeled by a simulation (line).

channel. Since continuous transport is necessary to measure $\langle\Delta x\rangle$, the experiment was performed in a channel with large reservoirs attached to both ends but otherwise identical geometry as in Fig. 3. For a given $T_{+}$, the mean displacement $\langle\Delta x\rangle$ of monodisperse $3.0 \mu \mathrm{m}$ colloids was measured after 100 cycles. This procedure was repeated for values of $T_{+}$ranging from $1 \mathrm{~s}$ to $78 \mathrm{~s}$. The time $T_{-}$was set to $T_{-}=T_{+}+20 \mathrm{~s}$, which was always sufficient for the particles to fully reach the small saw teeth. Fig. 4 shows the mean displacement $\langle\Delta x\rangle$ in units of $L$ as a function of the normalized interval $T_{+} / t_{\text {cross. }}$.

The diamonds represent experimental data of a highly diluted colloid suspension, i.e., in average about one particle per sawtooth, while the line represents data from Brownian dynamics simulations of a single particle (see ESI $\dagger$ ). For both experiment as well as simulation, the mean displacement $\langle\Delta x\rangle$ is negative for small values of $T_{+}$, i.e., particles are transported to the left interacting with the small profile only ( $c f$. the blue particle in Fig. 1). When particles reach the long profile for $T_{+} \approx t_{\text {cross, }}$, the mean displacement rapidly changes its sign as the influence of the large profile increases, as predicted. For larger values of $T_{+}$, the mean displacement approaches a maximal value as the particles always reach both minima ( $c f$. the red particle in Fig. 1). The experimental results for $T_{+} / t_{\text {cross }}>1.3$ are reproduced remarkably well by the simulation, which does not contain any fit to the experimental data. In particular, the point of direction reversal, which is important for the separation concept, is precisely reproduced. For smaller $T_{+}$, minor deviations result from the rotation mechanics, as described in the ESI $\dagger$.

We estimated the separation time in a system downscaled by a factor 10 while keeping the dimensionless parameters of the underlying Langevin Equation constant (see ESI† for details). Particles with diameter $300 \mathrm{~nm}$, for instance large macromolecules, including proteins and nucleic acids, or viruses, might be inserted into a ratchet device with $L=6 \mu \mathrm{m}$, which can be fabricated by means of standard e-beam lithography. With the time intervals being adjusted to the decreased length scales, the complete separation process of 450 cycles is remarkably reduced to less than 1 minute. This is an appealing time scale for the application in analytic devices and notably less time than required by conventional techniques, such as gel-electrophoresis. However, the presented gravitational driving restricts further downscaling, since Brownian motion overweighs sedimentation for particles smaller than $\sim 1 \mu \mathrm{m}$, thereby eventually impedes any directed transport. Hence, the implementation of stronger driving forces such as electric forces is unavoidable for smaller devices. In principle, the application of electric fields by electrodes parallel to the channel poses a technical rather than a conceptual problem. The electrodes need to be electrically insulated, in order to avoid electrolytic currents and dielectric heating of the dispersion. Still, the mutual influence of an electric field and dielectric channel walls raises unresolved challenges, ${ }^{25-27}$ so further investigation is needed.

In the following, we give a short overview of the device performance and prospects for the applications in lab-on-a-chip devices. The resolution in terms of purity of the output is fundamentally high and restricted only by channel width $d$ and available time. A large channel width can outweigh even a slight difference in the velocity of distinct types of particles. The only preconditions are a sufficiently low concentration of particles, i.e., not more than a few particles per saw tooth, and weak interaction between particles and the channel walls. Our device can then separate multiple types of particles in two fractions, provided they exhibit distinct drift velocities. The separation threshold is adjustable on the fly by variation of the frequency (i.e., the duration $T_{+} / t_{\text {cross }}$ ) of the external force. This provides a range of interesting applications. For example, a channel as in Fig. 1 can be loaded with the dispersion at one end and the other end can serve as output. In this way, the device can operate in different modes depending on the frequency of the driving force.

For high frequencies (i.e., short $T_{+} / t_{\text {cross }}$ ), it would act as a closed valve, whereas for low frequencies it would allow throughput of the dispersed particles. At medium frequencies, a multi-particle dispersion would be split, so that only faster particles were transported. Thus, a row of parallel channels results in an artificial membrane with adjustable permeability, combining separation as well as control functionality. The relatively low throughput caused by the high dilution, as mentioned above, can to some extent be compensated by such parallelization. Another method of feeding the device would be from above, i.e., perpendicular to the separation plane, so the particles would simply sediment into the center of a channel for processing. Depending on the frequency of the external force, the latter setup can either be used as a continuous separator or adjustable T-switch, in which the input is pumped to one of both channel outputs.

In a different configuration, the device may be used to split a multiparticle dispersion into several fractions. By loading the channel with dispersion from one end and lowering the frequency in discrete steps, particles of gradually lower velocity would be transported, thereby achieving velocity resolution over time. In order to attain simultaneous separation of multiple particle types, several separators might be connected in a row, each one featuring either a larger channel width $d$ or a larger frequency $1 / T_{+}$. Suspensions containing multiple particle types would split in such a device, with particles of specific drift speeds assembling at the interfaces between different channel segments, from where they can be collected.

\section{Conclusions}

In conclusion, we presented a proof of concept for a new class of ratchet-based separation devices, which provide binary output with high selectivity. The presented device has a size of about $0.1 \mathrm{~mm}^{2}$, which is at least one order of magnitude smaller than previously presented separation devices. This qualifies the presented separator for the integration in microfluidic analytic devices. Despite this remarkable size reduction, the results in this article only mark the upper bound for possible length scales. Through a scaling argument we demonstrated that the process is accelerated when applied at smaller length scales, due to the diffusion-based separation 
mechanism. However, an electric driving force is necessary for downscaling. Once driven electrically, such devices will add versatile separation functionality to lab-on-a-chip devices that could find widespread applications. Apart from their size, prominent characteristics of such devices would be the on the fly adjustable separation threshold, possible parallelization and the combination of separation and control features.

\section{Acknowledgements}

We gratefully thank Paul Leiderer (Konstanz University) and Hans Joachim Schöpe (Mainz University) for fruitful discussions and equipment. Support for this work was provided by BadenWürttemberg Stiftung, the German Research Foundation (DFG) and by the Center for Applied Photonics Konstanz.

\section{Notes and references}

1 J. Han, J. Fu and R. B. Schoch, Lab Chip, 2008, 8, 23-33.

2 Y. C. Lim, A. Z. Kouzani and W. Duan, Microsystem TechnologiesMicro-and Nanosystems-Information Storage and Processing Systems, 2010, vol. 16, pp. 1995-2015.

3 D. Mark, S. Haeberle, G. Roth, F. von Stetten and R. Zengerle, Chem. Soc. Rev., 2010, 39, 1153-1182.

4 N. Pamme, Lab Chip, 2007, 7, 1644-1659.

5 M. Yamada and M. Seki, Anal. Chem., 2006, 78, 1357-1362.

6 J. Fu, R. B. Schoch, A. L. Stevens, S. R. Tannenbaum and J. Han, Nat. Nanotechnol., 2007, 2, 121-128.

7 N. Xia, T. P. Hunt, B. T. Mayers, E. Alsberg, R. M. Westervelt and D. E. Ingber, Biomed. Microdevices, 2006, 8, 299-308.
8 A. Nilsson, F. Petersson, H. Jönsson and T. Laurell, Lab Chip, 2004 4, 131-135.

9 M. P. MacDonald, G. C. Spalding and K. Dholakia, Nature, 2003 426, 421-424

10 M. Smoluchowski, Phys. Z., 1912, 13, 1069-1080.

11 R. P. Feynman, R. B. Leighton and M. Sands, The Feynman Lectures on Physics, Addison-Wesley, Reading, MA, 1966.

12 M. Magnasco, Phys. Rev. Lett., 1993, 71, 1477-1481.

13 P. Hänggi, Rev. Mod. Phys., 2009, 81, 387-442.

14 R. D. Astumian, Science, 1997, 276, 917-922.

15 D. Ertas, Phys. Rev. Lett., 1998, 80, 1548-1551.

16 T. A. J. Duke and R. H. Austin, Phys. Rev. Lett., 1998, 80, 1552-1555.

17 I. Derenyi and R. D. Astumian, Phys. Rev. E: Stat. Phys., Plasmas, Fluids, Relat. Interdiscip. Top., 1998, 58, 7781-7784.

18 C. Kettner, P. Reimann, P. Hänggi and F. Müller, Phys. Rev. E: Stat. Phys., Plasmas, Fluids, Relat. Interdiscip. Top., 2000, 61, 312-323.

19 J. Rousselet, L. Salome and A. Ajdari, Nature, 1994, 370, 446-448.

20 A. van Oudenaarden and S. G. Boxer, Science, 1999, 285, 1046-1048.

21 C.-F. Chou, O. Bakajin, S. W. P. Turner, T. A. J. Duke, S. S. Chan, E. C. Cox, H. G. Craighead and R. Austin, Proc. Natl. Acad. Sci. U. S: A., 1999, 96, 13762-13765.

22 S. Matthias and F. Müller, Nature, 2003, 424, 53-57.

23 I. W. Hamley, Introduction to Soft Matter, Wiley, Chichester, 2007.

24 A. Grimm, H. Stark and J. R. C. van der Maarel, Phys. Rev. E: Stat, Nonlinear, Soft Matter Phys., 2009, 79, 061102.

25 Z. Li and G. Drazer, Phys. Rev. Lett., 2007, 98, 050602.

26 R. H. Austin, Appl. Phys. A: Mater. Sci. Process., 2002, 75, 279-284.

27 L. Huang, P. Silberzan, J. Tegenfeldt, E. Cox, J. Sturm, R. Austin and H. Craighead, Phys. Rev. Lett., 2002, 89, 178301.

28 D. C. Duffy, J. C. McDonald and O. J. A. Schueller, Anal. Chem. 1998, 70, 4974-4984.

29 A. Grimm, Doctoral thesis, National University of Singpore, 2010.

30 P. E. Kloeden and E. Platen, Numerical Solution of Stochastic Differential Equations, Springer, Berlin, Heidelberg, 1992. 